\title{
Current day mass loss rate for Luminous Blue Variable IRAS 18576+0341
}

\author{
G. Umana ${ }^{1,2}$, C. S. Buemi ${ }^{2}$, C. Trigilio ${ }^{2}$, and P. Leto ${ }^{2}$ \\ 1 INAF - Osservatorio Astrofisico di Catania, via S. Sofia 78, 95123 Catania Italy \\ 2 INAF - Istituto di Radioastronomia Sezione di Noto, CP 141, 96017 Noto (SR), Italy \\ e-mail: g.umana@ira.inaf.it
}

Received 23 February 2005 / Accepted 13 May 2005

\begin{abstract}
In this letter we report on the first set of multi-frequency and high angular resolution radio observations of IRAS 18576+0341. The radio observations have revealed an extended, asymmetric and quite structured radio nebula and allowed us to locate the central core of this highly obscured new galactic Luminous Blue Variable. From the analysis of radio properties of IRAS $18576+0341$ estimates of important physical parameters of the central star have been determined. In particular, an effective temperature of $T_{\text {eff }} \sim(2.6 \pm 0.2) \times 10^{4} \mathrm{~K}$, corresponding to a B0-B0.5 supergiant, has been derived. The most notable result is the determination of the current day mass-loss $\left(\dot{M}=3.7 \times 10^{-5} M_{\odot} \mathrm{yr}^{-1}\right)$, which is of particular importance since mass-loss rate determination from radio observations appears to be a more reliable method compared to the others based on different diagnostics.
\end{abstract}

Key words. circumstellar matter - stars: winds, outflows - stars: mass-loss - radio continuum: stars stars: individual: IRAS 18576+0341, AFGL 2298

\section{Introduction}

A Luminous Blue Variable (LBV) is an evolved, luminous massive star which is characterized by unsteady, high massloss rate $\left(\dot{M}=10^{-3}-10^{-4} M_{\odot} \mathrm{yr}^{-1}\right)$. Stars belonging to this class undergo giant eruptions, leading to the formation of extended circumstellar nebulae, and are characterized by strong spectral and photometric variability. They are believed to be in the short transition phase of massive star evolution towards the Wolf-Rayets stage (Humphreys \& Davidson 1994). Typical LBVs are $\eta$ Carinae and P Cyg.

LBVs are very rare; up to now less than 10 objects are known in our Galaxy. This is probably related to the very short timescales involved in this phase of stellar evolution $\left(\sim 10^{4} \mathrm{yr}\right)$ and to the presence of thick circumstellar envelope that makes observations of the central object difficult. Among new LBVs candidates there is the infrared source IRAS $18576+0341$ that was firstly classified as a Planetary Nebula on the basis of its infrared colors (Garcia-Lario et al. 1997). More recently, Ueta et al. (2001) mapped the source at 10.3 and $18.0 \mu \mathrm{m}$ and reported the presence of an extended dusty circumstellar shell, whose morphology is consistent with a central object that have experienced strong mass-loss. This, together with the modelling of the spectral energy distribution (SED), which supported an extremely bright $\left(L_{*}=2.5 \times 10^{6} L_{\odot}\right)$ central object, led the authors to suggest that IRAS $18576+0341$ could be a LBV.

The source has been definitively classified as a new LBV by Pasquali \& Comeron (2002) and by Clark et al. (2003), on the basis of the observed strong photometric and spectroscopic variability and of its position in the HR diagram.

Radio observations of LBVs are particularly important because their ability to penetrate the thick dusty envelopes and thus to probe the gaseous component lying inside them. Moreover, radio observations provide a powerful tracer for $\dot{M}$, because less assumptions than those required by other $\dot{M}$ determination methods are necessary. A good $\dot{M}$ determination is very important since the observed variability of mass-loss rate and its relation to changes in $T_{\text {eff }}$ (or radius) during the cyclic spectral and photometric variations of LBVs is still under debate, mainly because of the paucity of available measurements. So far, P Cyg is the only LBV of the northern hemisphere to be studied in detail in the radio, as the other radio emitting LBVs are all in southern hemisphere, where radio interferometers with angular resolution of subarcsec scale are not available. In this letter we report on the first set of multifrequency and high spatial resolution VLA radio observations of IRAS $18576+0341$. 


\section{Observations}

We observed IRAS $18576+0341$ with the $\mathrm{VLA}^{1}$ at six frequencies, namely 1.4 ( $L$-band), 4.8 ( $C$-band), 8.4 ( $X$-band), 14.9 ( $U$-band), 22.0 ( $K$-band) and 43.0 ( $Q$-band) $\mathrm{GHz}$, with a bandwidth of $100 \mathrm{MHz}$.

The observations were carried out in three different days, namely on April 18, 20 and 22, 2004. The array was in C configuration, providing a typical beam size of $\sim 12.5^{\prime \prime}, \sim 3.9^{\prime \prime}$, $\sim 2.3^{\prime \prime}, \sim 1.2^{\prime \prime}, \sim 0.9^{\prime \prime}$ and $\sim 0.47^{\prime \prime}$ at 1.4, 4.8, 8.4, 14.9, 22 and $43 \mathrm{GHz}$ respectively.

For the lower frequencies $1824+107$ was used as phase calibrator, while for $K$ and $Q$-band the closer $1851+005$ was preferred. For all the observed band the flux density scale was determined by observing $3 \mathrm{C} 48$.

The data processing was performed using the standard programs of the NRAO Astronomical Image Processing System (AIPS). The mapping process was performed by using the UNIFORM weighting and the dirty map was CLEANed down as close as possible to the theoretical noise. Observations were obtained in different dates. We therefore, for each band, firstly mapped the data relative at each single day. After checking that short term variability was not present in our data, we concatenated the data of each frequency in a single data set. This allowed for a better signal to noise ratio. The noise level (rms) in the maps was estimated by analyzing an area on the map, whose dimension is of the order of more than $100 \theta_{\mathrm{syn}}^{2}$, away from the phase center and free from evident radio sources. At each frequency, the map rms resulted to be consistent with the expected theoretical noise.

We detected IRAS $18576+0341$ at all the 6 observed frequencies as an extended nebula. The total radio flux density associated to the source has been derived by summing the contribution of all the pixels contained in the radio source (AIPS task IMSTAT).

\section{The radio properties}

IRAS $18576+0341$ is an extended radio source, however, the limited angular resolution of the $\mathrm{C}$ configuration prevented the identification of the core component at lower frequencies. Therefore at those frequencies only the total flux density and an estimate of the overall dimension of the source could be gathered. From the $1.4 \mathrm{GHz}$ map an estimate of the dimension of the extended nebula can be derived, which is of the order of $\leq 10^{\prime \prime}$. An interferometer is blind to structures whose angular dimensions are larger then the shortest baselines. At $0.7 \mathrm{~cm}$ the VLA-C is sensitive to structures up to $40^{\prime \prime}$. Therefore, in the conservative hypothesis that the overall dimension of the extended nebula is the same at all the frequencies, we can be quite confident that all of the flux from the nebula is detected, even at $0.7 \mathrm{~cm}$.

At the higher frequencies a compact, slightly resolved source is evident, embedded in a structured extended ionized

${ }^{1}$ The Very Large Array is a facility of the National Radio Astronomy Observatory which is operated by Associated Universities, Inc. under cooperative agreement with the National Science Foundation.

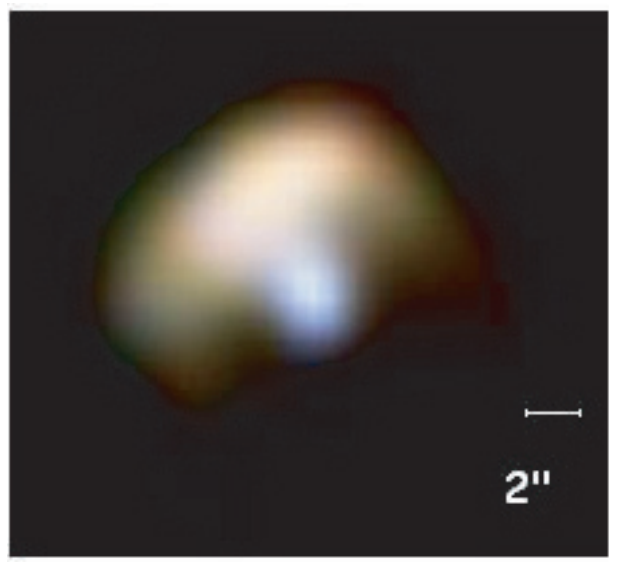

Fig. 1. Composite image obtained by superposition of radio images at 43, 22 and $14.9 \mathrm{GHz}$. Color coding is: $14.9 \mathrm{GHz}$ red, $22.0 \mathrm{GHz}$ green and $43.0 \mathrm{GHz}$ blue.

shell. The same kind of morphology has been observed in other LBVs. Figure 1 shows the composite image obtained by superposition of radio images at 43,22 and $14.9 \mathrm{GHz}$ after degradation to a common angular resolution of $0.4\left(\theta_{F W H M} / 3\right)$ at $14.9 \mathrm{GHz}$. The total $K$-band $(22 \mathrm{GHz})$ and $Q$-band $(43 \mathrm{GHz})$ maps are shown in Figs. 2a and b. Most of radio flux is coming from the extended, highly clumpy nebula with negligible contribution from the compact one, that, however, becomes more important as the frequency increases.

To quantify the contribution of the two components (central object and nebula) to the observed flux density we have then reconstructed the radio spectrum (central object plus nebula).

\subsection{The core component}

From the higher frequency maps, where the angular resolution allows us to clearly distinguish the compact component, the position, flux density and angular size of the central source have been derived by fitting a two dimensional Gaussian brightness distribution to the map. The same procedure was applied to the $K$ and $U$ band data, by using as a fixed parameter of the fit the $Q$-band source position, namely $\alpha=19^{\mathrm{h}} 00^{\mathrm{m}} 10^{\mathrm{s}} .92$, $\delta=+03^{\circ} 45^{\prime} 47^{\prime \prime} .1$.

The results obtained for the central core component plus their uncertainties are given in Table 1 . At each frequency, the error associated to the flux density estimation $F_{v}$ is 3 times the rms in the map.

In Fig. 3 the spectrum of the compact core is shown. A stellar wind behavior is evident, which is characterized by a $0.8 \pm 0.1$ spectral index $\left(v^{\alpha}\right)$ obtained from a weighted linear fit of 15, 22 and $43 \mathrm{GHz}$ measurements.

\subsection{The extended nebula}

To get an estimate of the radio flux density due only to the extended nebula, at the higher frequencies we directly subtracted, from the total flux density, the contribution of the core component, as derived by the Gaussian fit. For the lower frequencies 

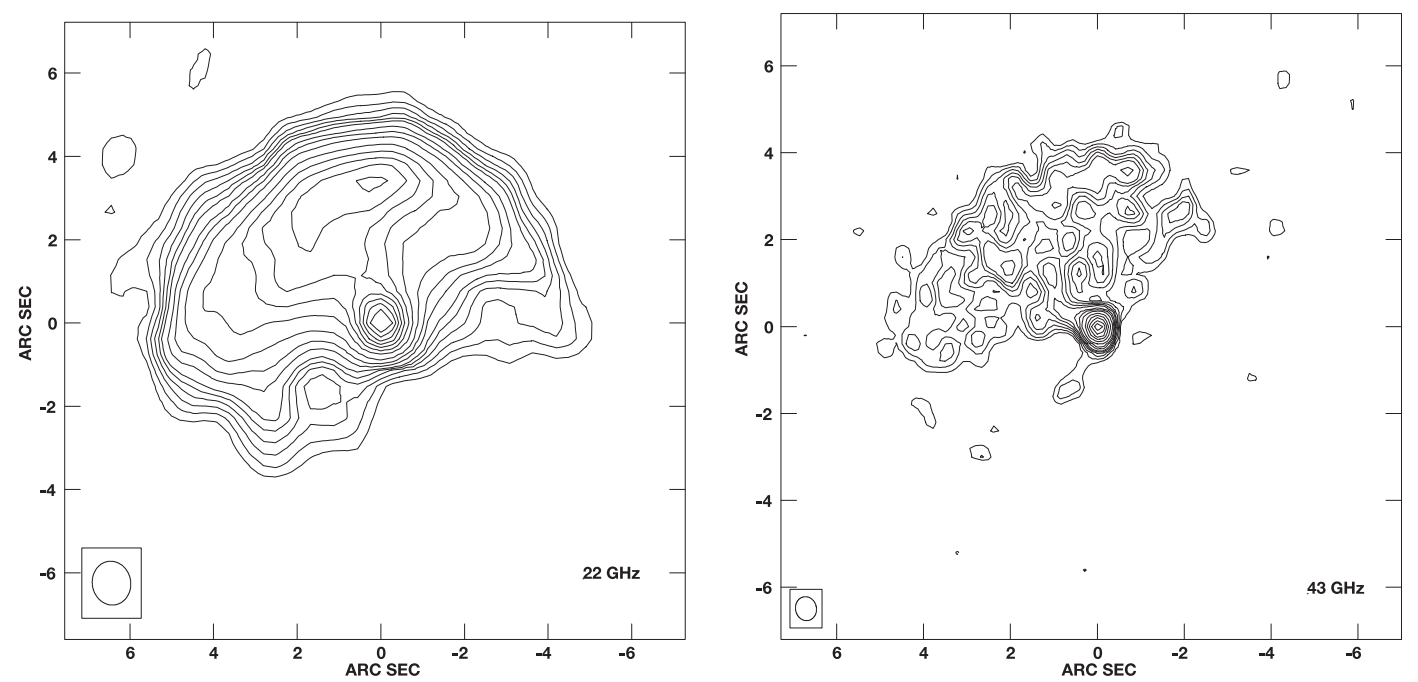

Fig. 2. a) Contour plot of $1.3 \mathrm{~cm}$ image. Levels are $-3,3,4,5,6,7,8,10,12,15,20,25,30,40,45,50,60,70,80,90$ times $0.09 \mathrm{mJy} / \mathrm{beam}$; b) Contour plot of $0.7 \mathrm{~cm}$ image. Levels are $-3,3,4,5,6,7,8,10,12,15,20,25,30,40,45,50,60,70,80,90$ times $0.15 \mathrm{mJy} / \mathrm{beam}$.

Table 1. Radio properties of the Nebula and the core. At lower frequencies, values of flux density of the core component are extrapolations of the high frequency trend.

\begin{tabular}{c|ccc|cc}
\hline \hline & \multicolumn{3}{|c|}{ Nebula } & \multicolumn{2}{c}{ Core component } \\
\hline $\begin{array}{c}\text { Band } \\
{[\mathrm{GHz}]}\end{array}$ & $\begin{array}{c}\text { Flux density } \\
{[\mathrm{mJy}]}\end{array}$ & $\begin{array}{c}\text { Size }(F W H M) \\
{[\operatorname{arcsec}]}\end{array}$ & $\begin{array}{c}\text { Orientation (major axis) } \\
{[\mathrm{deg}]}\end{array}$ & $\begin{array}{c}\text { Flux density } \\
{[\mathrm{mJy}]}\end{array}$ & $\begin{array}{c}\text { Size } \\
{[\operatorname{arcsec}]}\end{array}$ \\
\hline 1.4 & $83 \pm 2$ & - & - & 0.6 & - \\
4.8 & $100.9 \pm 0.5$ & $5.67 \times 4.02$ & $106.4^{\circ}$ & 1.5 & - \\
8.4 & $95.0 \pm 0.2$ & $5.71 \times 3.93$ & $109.8^{\circ}$ & 2.1 & - \\
14.9 & $87.9 \pm 0.4$ & $5.72 \times 3.89$ & $113^{\circ}$ & $3.1 \pm 0.4$ & - \\
22 & $78.0 \pm 0.3$ & $5.82 \times 3.69$ & $113.8^{\circ}$ & $4.1 \pm 0.3$ & $0.28 \times 0.20$ \\
43 & $73.8 \pm 0.5$ & $5.99 \times 3.75$ & $117^{\circ}$ & $6.2 \pm 0.5$ & $0.23 \times 0.16$ \\
\hline
\end{tabular}

the contribution of the core component was evaluated from the extrapolation of the stellar wind spectrum down to $1.4 \mathrm{GHz}$.

The results obtained for the extended component are also reported in Table 1 and shown in Fig. 3. The optically thick spectrum $\left(F_{v} \propto v^{2}\right)$ and optically thin spectrum $\left(F_{v} \propto v^{-0.1}\right)$ of a free-free emitting radio sources are also shown for comparison.

IRAS $18576+0341$ has been previously observed in 1991 by Becker et al. (1994) during a large $5 \mathrm{GHz}$ survey conducted with the VLA. They measured a total flux density of $78 \pm 7.5 \mathrm{mJy}$. If we compare this results with our $5 \mathrm{GHz}$ measurements, even considering the poorer angular resolution and sensitivity of Beckers' data, it appears that the source is variable at least on timescale of order of years.

\section{Discussion}

\subsection{The Nebula properties}

The most striking characteristics of the radio nebula is its asymmetry, as the nebula lying all well in the N-E part with respect to the central object. A certain degree of asymmetry was also evident in the mid-IR map obtained by Ueta et al. (2001), where a southern component, which contributes to the $25 \%$ of the total mid-IR emission is present. This southern component is not present in any of the radio maps, where the first contour level, of 3 times the map rms, corresponds to $0.1 \%$ of the total radio emission. The fact that this asymmetry in the nebula is clearly visible in the radio, which is not affected by absorption, leads to exclude any effect due to absorption by dust.

Such an asymmetry is present in other LBVs and, in particular, White et al. (2000), in order to explain it pushed forward the hypothesis of a unseen binary conpanion, which ionizes part of the envelope, giving origin to the unbalanced ionized region. While the explanation of such a morphology is beyond the scope of this letter and needs further, more focused observations, it appears that the binary model evocated by White et al. (2000) in the case of HR Car may be not valid in the present situation as the temperature map obtained from mid-IR observations by Ueta et al. (2001) indicates a single, central, heating source.

From the optically thin radio flux it is possible to derive the total ionized mass of the nebula (Gatheir et al. 1983). In the hypothesis of a pure hydrogen nebula at $10 \mathrm{kpc}$, with a electron temperature of $10^{4} \mathrm{~K}$, an average angular radius of $5^{\prime \prime}$, we derive from the flux density at $22 \mathrm{GHz}$ a total ionized mass 


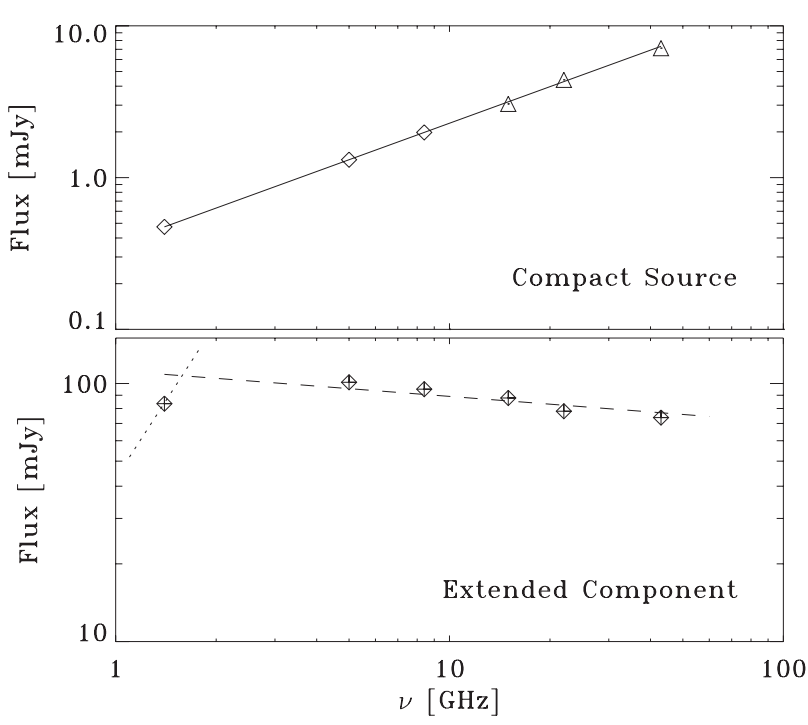

Fig. 3. Radio spectra of the central object and of the extended nebula. For the compact object, triangles indicate the measurements used in the fit to derive the spectral index. Diamonds indicate extrapolations of such a fit to the lower frequencies. For the extended nebula, the optically thick spectrum (dotted line) and optically thin spectrum (dashed line) of a free-free emitting radio sources are shown for comparison.

of $\sim 2 M_{\odot}$. This value depends on the value of volume filling factor of the nebula, which has been assumed to be 1 .

Ueta et al. (2001) provide an estimate of total mass of dust of $\sim 0.1 M_{\odot}$, which was computed assuming a toroidal morphology for the dust emitting region, with a radius of $\sim 14^{\prime \prime}$. Therefore, a dust emitting region, whose dimensions are comparable to those of the radio emitting nebula, would contain a total mass of dust of $4.2 \times 10^{-3} M_{\odot}$, which implies a dust to gas ratio of $2 \times 10^{-3}$. This value, however, has to be considered as an upper limit, because radio observations trace only the ionized gas. The difference from the canonical value of $\sim 10^{-2}$ may be attributable to the fact that values derived by Ueta et al. (2001) are strongly model depended.

\subsection{The $T_{\text {eff }}$ of the central object}

The nebular radio spectrum shows that, starting from $4.8 \mathrm{GHz}$, the extended nebula is optically thin. It is, therefore, possible to derive the excitation parameter $\left(U_{\text {exc }}\right)$ required to account for the measured radio flux:

$U_{\mathrm{exc}}=13.3\left(v^{0.1} T^{0.35} D_{\mathrm{kpc}}^{2} F_{v}\right)^{\frac{1}{3}} \quad \mathrm{pc} \mathrm{cm}^{-2}$

where $F_{v}$ is the optically thin radio flux density, expressed in Jy, at the observing frequency $v$ (in $\mathrm{GHz}$ ); $T$ is the nebula temperature, expressed in $10^{4} \mathrm{~K}$, and $D_{\mathrm{kpc}}$ is the source distance in kpc.

If the nebula is ionization bounded, this parameter can be related to the radiation field of the ionizing source, namely to the number of ionizing photons, with wavelengths shorter than the Lyman limit, emitted by the central star. Following the definition by Spitzer (1978) we have:

$L_{\mathrm{uv}}=1.23 \times 10^{56} \beta U_{\mathrm{exc}}^{3}$ photons s $^{-1}$ where $\beta$ is the hydrogen recombination coefficient summed over all levels above the ground level $\left(\sim 3 \times 10^{-13} \mathrm{~cm}^{3} \mathrm{~s}^{-1}\right.$ for $T \sim 10^{4} \mathrm{~K}$ ).

By combining Eq. (1) with Eq. (2), assuming a nebular temperature of $T \sim 10^{4} \mathrm{~K}$ and a distance of $10 \mathrm{kpc}$ (Ueta et al. 2001), from the radio flux density measured at $22 \mathrm{GHz}$, we derive a number of ionizing photons of $L_{\mathrm{uv}} \sim 8.95 \times$ $10^{47}$ (photons s ${ }^{-1}$ ). This is the flux of Lyman continuum photons of a B0-B0.5 supergiant and corresponds to an effective temperature of $T_{\text {eff }} \sim(2.6 \pm 0.2) \times 10^{4} \mathrm{~K}$ (Panagia 1973).

Spectral characteristics of IRAS $18576+0341$ are very similar to those of other galactic LBVs classified as Ofpe/WN9 and WN9-11 as pointed out by Pasquali \& Comeron (2002). This would imply a $T_{\text {eff }}$ in the range $(2.8 \pm 0.7) \times 10^{4} \mathrm{~K}$. On the other hand, Clark et al. (2003) performed a modelling of the near-IR spectrum of IRAS $18576+0341$, deriving a $T_{\text {eff }}$ between 1.3 and $1.5 \times 10^{4} \mathrm{~K}$. Similar values $\left((1.5 \pm 0.6) \times 10^{4} \mathrm{~K}\right)$ were obtained by Ueta et al. (2001) by modelling the dusty shell. Our result is in between these extremes but it has to be taken with some caution as ionizing fluxes for hot stars have been derived from stellar structure and atmospheric models of normal stars while properties of LBVs show many similarities but also several differences with those of normal blue supergiants (Crowther 1997).

\subsection{The current day mass-loss rate}

If we assume that the central radio object is coincident with the LBV we can derive, from the radio data, its actual mass-loss.

Standard formulas for thermal radio emission from an expanding wind have been derived by Panagia \& Felli (1975). They showed that the observed radio flux $\left(F_{v}, \mathrm{mJy}\right)$ is related to the dynamical parameters of the wind such as mass-loss rate $(\dot{M})$ and the terminal velocity of the wind $\left(v_{\infty}, \mathrm{km} \mathrm{s}^{-1}\right)$. It is therefore possible to estimate the mass-loss by using the relation:

$\dot{M}=6.7 \times 10^{-4} v_{\infty} F_{v}^{3 / 4} D_{\mathrm{kpc}}^{3 / 2}\left(v \times g_{\mathrm{ff}}\right)^{-0.5} \quad M_{\odot} \mathrm{yr}^{-1}$

where full ionization and cosmic abundances have been assumed. The free-free Gaunt factor $\left(g_{\mathrm{ff}}\right)$ is approximated with $g_{\mathrm{ff}}=9.77\left(1+0.13 \log \frac{T^{3 / 2}}{v}\right)$ (Leitherer \& Robert 1991).

From the radio flux density observed at $43 \mathrm{GHz}$, assuming as stellar wind velocity a value of $v \sim 200 \mathrm{~km} \mathrm{~s}^{-1}$, (Crowther 1997), a temperature of the wind of $10^{4} \mathrm{~K}$ and a distance of $10 \mathrm{kpc}$ (Ueta et al. 2001), we derive a mass loss rate of $\dot{M}=3.7 \times 10^{-5} M_{\odot} \mathrm{yr}^{-1}$. This value is consistent with previous evaluations obtained by different authors using different techniques (Leitherer 1997). In particularly, it is consistent with values reported by Clark et al. (2003), who estimated, for this object, a variation of mass loss rate from $0.5 \times 10^{-4}$ to $1.2 \times$ $10^{-4} M_{\odot} \mathrm{yr}^{-1}$ in less than one year, associated to an increment of $T_{\text {eff }}$ from $1.25 \times 10^{4}$ to $1.5 \times 10^{-4} \mathrm{~K}$. Changing mass loss rate is not surprising for LBVs, which shows a wide range of behavior as a function of effective temperature (Leitherer 1997; Stahl et al. 2001). Vink \& de Koter (2002) suggest that the observed behavior can be explained as a consequence of the changes in the efficiency of line driving resulting from the recombination/ionization Fe VI/III and Fe III/II, the second one 
predicted to occur for $T_{\mathrm{eff}} \simeq 1.5 \times 10^{4} \mathrm{~K}$, that is in the observed range of variability of $T_{\text {eff }}$ for IRAS $18576+0341$.

Finally, we may compare the value of current mass loss with those derived from the properties of the ejected dust shell, which is the remnant of the previous eruption episodes experienced by IRAS $18576+0341$ in the past. From the model of the dusty shell, Ueta et al. (2001) derived a value of mass loss, which is an order of magnitude lower than the current values. Since the difference is not attributable to an assumed too low dust to gas ratio (see Sect. 4.1), it appears that IRAS $18576+0341$ is in the process of making another LBV shell.

\section{Conclusions}

We have obtained the first high resolution, multi-frequency radio images of the newly recognized galactic LBV IRAS $18576+0341$, which have revealed the core compact component, assumed to be coincident with the LBV, embedded in a more extended, highly structured nebula.

The radio properties of the extended nebula has been compared with those of the mid-IR dust shell and the derivation of the total mass of ionized gas allowed us to determine the dust to gas ratio in the nebula, which appears to be at least one order of magnitude smaller than the value observed in the interstellar medium.

The detection of radio emission from a stellar wind associated to the central core of IRAS $18576+0341$ have provided the possibility to get accurate estimates of the current day $\dot{M}$.

Successive monitoring of the high frequency radio flux density of IRAS $18576+0341$ together with more near-IR spectroscopic observations will allow us to derive relevant stellar parameters, i.e. $T_{\text {eff }}$ and $\dot{M}$, to determine characteristic timescale of their variability and to establish any kind of relation among them.

Acknowledgements. We wish to thank the anonymous referee for his/her very useful suggestions. This research has made use of the SIMBAD database, operated at CDS, Strasbourg, France.

\section{References}

Becker, R. H., White, R. L., Helfland, D. J., \& Zoonematkermani, S. 1994, ApJS, 91, 347

Clark, J. S., Larionov, V. M., Crowther, P. A., Egan, M. P., \& Arkharov, A. 2003, A\&A, 403, 653

Crowther, P. A. 1997, ASP Conf. Ser., 120, 51

Garcia-Lario, P., Manchado, A., Pych, W., \& Pottasch, S. R. 1997, A\&AS, 126, 479

Gathier, R. 1987, A\&AS, 71, 245

Humphreys, R. M., \& Davison, K. 1994, PASP, 106, 1025

Leitherer, C. 1997, ASP Conf. Ser., 120, 58

Leitherer, C., \& Robert, C. 1991, ApJ, 377, 629

Pasquali, A., \& Comeron, F. 2002, A\&A, 382, 1005

Panagia, N. 1973, AJ, 78, 929

Panagia, N., \& Felli, M. 1975, A\&A, 39, 1

Spitzer, L. 1978, Physical Process in the Interstellar Medium (Wiley-Interscience Publication)

Stahl, O., Jankovics, I., Kovács, J., Wolf, B., et al. 2001, A\&A, 375, 54

Ueta, T., Meixner, M., Dayal, A., et al. 2001, ApJ, 548, 1020

Vink, J. S., \& de Koter, A. 2002, A\&A, 393, 543

White, S. M. 2000, ApJ, 539, 851 